\title{
Rectal cap polyposis: a rare cause of rectal bleeding, anaemia and mucoid diarrhoea
}

\begin{abstract}
Cap Polyposis (CP) is an under recognized form of nonneoplastic inflammatory polyps, with a distinct cap of granulation tissue. This is an interesting case of a young male presented with bleeding per rectum, mucoid diarrhoea and generalised anasarca. He underwent surgery for the rectal mucosal infiltration, improved thereafter and was managed as inflammatory bowel disease for three years, subsequently the disease again reappeared. He was finally diagnosed as rectal cap polyposis after repeat biopsy and was undergone polypectomy. There was significant improvement after the procedure and he was on regular followup. Awareness of this diagnosis is important as misdiagnosis can result in prolonged and inappropriate treatment.
\end{abstract}

Volume 10 Issue 4 - 2019

\author{
Bijay Misra,' Kedarnath Panda,' Prakash \\ Chandra Dalai,' Brundaban Nahak, ${ }^{2}$ Amit \\ Adya $^{3}$ \\ 'Department of Gastroenterology, Institute of Gastro and \\ Kidney Care Bhubaneswar, India \\ ${ }^{2}$ Department of Gastroenterosurgery, Institute of Gastro and \\ Kidney Care Bhubaneswar, India \\ ${ }^{3}$ Department of Pathology, KIMS Hospital Bhubaneswar, India
}

\author{
Correspondence: Bijay Misra, Department of \\ Gastroenterology, Institute of Gastro and Kidney Care \\ Bhubaneswar, Odisha, India.751015, \\ Emaildr_bijaymisra@yahoo.com
}

Received: July 27, 2017 | Published: July 08, 2019

\section{Introduction}

Cap Polyposis (CP) is an under recognized form of nonneoplastic inflammatory polyps, with a distinct cap of granulation tissue. Here we present a patient who was diagnosed with CP during the investigation of unexplained chronic long standing anaemia secondary to intermittent rectal bleeding and mucoid diarrhoea.

\section{Case report}

A twenty three year male presented with loose motion on and off, intermittent bleeding per rectum $(\mathrm{P} / \mathrm{R})$ since last 3years, bilateral pedal edema and scrotal swelling followed by generalized anasacra for 1year. The loose motion was 10 to 12 times per day, small in amount, associated with tenesmus and intermittent bleeding $\mathrm{P} / \mathrm{R}$, there was nocturnal symptoms and significant weight loss. Patient was having history of constipation earlier started before this episode associated with prolongs straining during defecation. Stool was occasionally mixed with fresh blood and there is no history of manual evacuation or enema use. During this episode he had significant weight loss despite normal appetite, developed generalized anasarca and scrotal swelling. No history of fever, joint pain, pain in eyes, skin ulcerations, hypertension, diabetes and tuberculosis in past. There was no history of substance abuse/allergy. General examination revealed pallor, generalized anasacra, ulcereated oral mucosa, lusterless skin and brittle hair. Gastrointestinal system revealed ascites, no mass abdomen. On per rectal examination there were lax anal sphincter and polypoidal mass lesions felt to the examining finger. On colonoscopy there is circumferential nodularity, erosions and narrowing from 5 to $12 \mathrm{~cm}$, biopsy came as tubular adenomatous changes with inflammation. Low anterior resection with transverse colon loop colostomy was done. Resected specimens do not show any evidence of malignancy. Colostomy closure was done after 4 month. Post operative period was uneventful. Patient again had symptoms after 6months, and develops generalized anasacra. He was managed with multiple albumin infusions. Colonoscopy examination done revealed lax anal sphincter, multiple yellowish white polypoidal lesions involving anorectal junction and lower rectum. Rest of the colonic mucosa up to terminal ileum appeared normal (Figure1). Gastroduodenoscopy was normal with negative rapid urease test for $\mathrm{H}$ Pylori. On biopsy there is mucosal ulcerations with granulation tissue, crypt dilatation and hyperplasia,dense lymphocytic and plasma cell infiltrations in lamina propia (Figures $2 \& 3$ ). It was consistent with rectal cap polyposis. Patient was managed conservatively and planned for endoscopic polypectomy. There were symptomatic improvement after the procedure and was on regular follow-up.

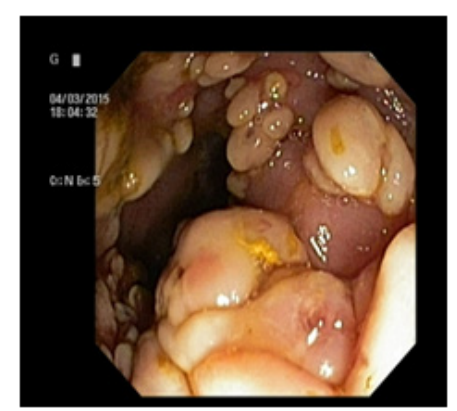

Figure I Colonoscopy revealed lax anal sphincter, multiple polypoidal lesions with normal intervening mucosa over rectum.

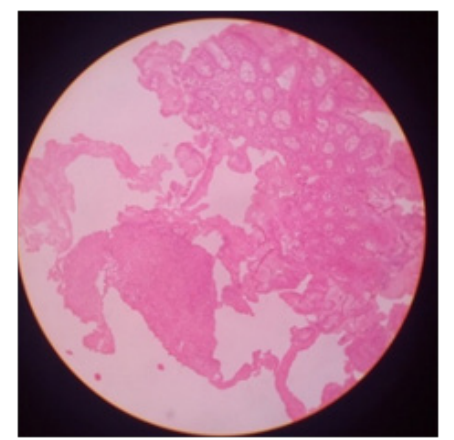

Figure 2 Low power photomicrograph $[\times 100] \mathrm{H}$ and E stain, showing colonic mucosal ulceration with granulation tissue. 


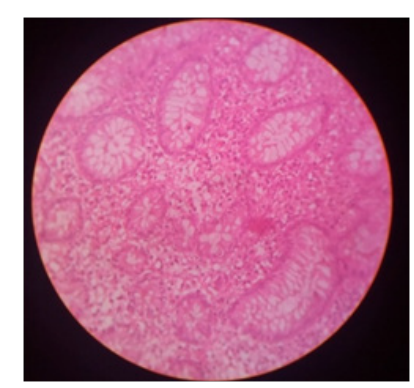

Figure 3 High power view [x400] H and E stain showing crypt dilatation and hyperplasia, dense lymphocytic and plasma cell infiltration in the lamina propria.

\section{Discussion}

Cap polyposis (CP) is poorly recognized form of non neoplasticcolonic polyps. These are inflammatory polyps with a distinct "cap" of granulation tissue over it. CP is often seen masquerading as chronic inflammatory bowel disease It usually affects people of age $>50$ years (range: 12-76years) with a female predominance. ${ }^{1}$ But our case is of a young male. The usual presentation were long standing anaemia, intermittent rectal bleeding, abdominal pain, tenesmus, hypoprotenemia due to protein losing enteropathy. ${ }^{2,3}$ Most common site rectosigmoid, also involve proximal colon, stomach and ileal pouch. Endoscopically, these are sessile polyps covered by a "cap" of fibrinopurulent exudates with normal intervening normal mucosa differentiates these from ulcerative colitis. These are not adenomatous, thereby familial adenomatous polyposis can be excluded. ${ }^{4}$ The exact aetiology of CP is not known however abnormal colonic motility and repeated trauma to the colonic mucosa caused by straining at defecation have been postulated as the initiating events. In this case patient had undergone surgery earlier in suspicicion of malignancy. After recurrence of lesion it was treated as inflammatory bowel disease. Finally it was diagnosed as case of rectal cap polyposis and offered endoscopic therapy. Multidisciplinary approach is needed in the management of patients with $\mathrm{CP}$ as this condition holds good long term prognosis. Conservative treatment modalities like avoidance of straining at defecation, use of metronidazole, infliximab, Helicobacter pylori eradication therapy and endoscopic polypectomy have been shown to be beneficial in some cases. ${ }^{5,6}$ Surgical resection should be reserved for patients with recurrence or for those who don't respond to conservative therapy.

\section{Conclusion}

Awareness of this diagnosis is important as misdiagnosis can result in prolonged and inappropriate treatment. Mucosal prolapse is the underlying mechanism in these cases. Rectal CP should be considered in the differential diagnosis of patients presenting clinically with long standing intermittent rectal bleeding and rectal polyposis on endoscopic examination. Endoscopic or surgical excision can be curative but recurrence is also common in these patients.

\section{Acknowledgments}

None.

\section{Conflicts of interest}

The authors declare there are no conflicts of interest related to the article.

\section{References}

1. Ng KH, Mathur P, Kumarasinghe MP, et al. Cap polyposis: Further experience and review. Dis Colon Rectum. 2004;47(7):1208-1215.

2. Campbell AP, Cobb CA, Chapman RW, et al. Cap polyposis: An unusual cause of diarrhoea. Gut. 1993;34(4):562-564.

3. Gallegos M, Lau C, Bradly DP, et al. Cap polyposis with proteinlosing enteropathy. Gastroenterol Hepatol (NY). 2011;7(6):415-420.

4. Mills ES. $5^{\text {th }}$ edn. Philadelphia: Lippin Cott Williams and Wilkins; 2010.

5. Takeshima F, Senoo T, Matsushima K, et al. Successful management of cap polyposis with eradication of Helicobacter pylori relapsing 15 years after remission on steroid therapy. Intern Med. 2012;51(4):435-439.

6. Kim ES, Jeen YT, Keum B, et al. Remission of cap polyposis maintained for more than three years after infliximab treatment. Gut Liver. 2009;3(4):325-328. 